\title{
Wound healing potential of human umbilical cord mesenchymal stem cell conditioned medium: An in vitro and in vivo study in diabetes-induced rats
}

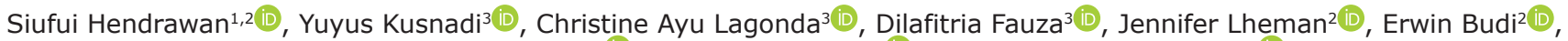 \\ Brian Saputra Manurung ${ }^{2}$, Hans Ulrich Baer ${ }^{4,5}$ (i) and Sukmawati Tansil Tan ${ }^{6}$ (i)
}

1. Department of Biochemistry and Molecular Biology, Faculty of Medicine, Tarumanagara University, 11440, Jakarta, Indonesia; 2. Tarumanagara Human Cell Technology Laboratory, Tarumanagara University, 11440, Jakarta, Indonesia; 3. Stem Cell Division, Stem Cell and Cancer Institute, PT. Kalbe Farma, Tbk., 10510, Jakarta, Indonesia; 4. Baermed, Centre of Abdominal Surgery, Hirslanden Clinic, 2501, Zürich, Switzerland; 5. Department of Visceral and Transplantation

Surgery, University of Bern, 3012, Bern, Switzerland; 6. Department of Dermatovenereology, Faculty of Medicine, Tarumanagara University, 11440, Jakarta, Indonesia.

Corresponding author: Siufui Hendrawan, e-mail: siufui@gmail.com

Co-authors: YK: yuyus.kusnadi@kalbe.co.id, CAL: christine.lagonda@gmail.com, DF: dilafitria.fauza@kalbe.co.id, JL: jenlheman@hotmail.com, EB: erwinblankk@gmail.com,BSM: manurung.brian@ymail.com,

HUB: hans.baer@baermed.ch, STT: dr.sukmawatitansilspkk@gmail.com

Received: 01-03-2021, Accepted: 08-07-2021, Published online: 17-08-2021

doi: www.doi.org/10.14202/vetworld.2021.2109-2117 How to cite this article: Hendrawan S, Kusnadi Y, Lagonda CA, Fauza D, Lheman J, Budi E, Manurung BS, Baer HU, Tan ST (2021) Wound healing potential of human umbilical cord mesenchymal stem cell conditioned medium: An in vitro and in vivo study indiabetes-induced rats, Veterinary World, 14(8): 2109-2117.

\begin{abstract}
Background and Aim: Human umbilical cord mesenchymal stem cells (hUC-MSCs) and its conditioned medium (CM) promote wound healing. This study investigated the wound healing potential of hUC-MSC CM in vitro and in vivo using diabetic animal models

Materials and Methods: The CM from hUC-MSC CM prepared under hypoxic conditions (hypoxic hUC-MSC) was evaluated for stimulating rat fibroblast growth, collagen production (in vitro), and wound healing in animal models (in vivo). An excision wound on the dorsal side of the diabetes-induced rats was established, and the rats were randomly divided into non-treatment, antibiotic, and hypoxic hUC-MSC CM groups. The cell number of fibroblasts and collagen secretion was evaluated and compared among the groups in an in vitro study. By contrast, wound size reduction, width of re-epithelialization, and the collagen formation area were assessed and compared among the groups in an in vivo study.

Results: CM under hypoxic conditions contained a higher concentration of wound healing-related growth factors. Hypoxic hUC-MSC CM could facilitate fibroblast cell growth and collagen synthesis, although not significant compared with the control group. Re-epithelialization and collagen production were higher in the hUC-MSC CM group than in the antibiotic and non-treatment groups.
\end{abstract}

Conclusion: Hypoxic hUC-MSC CM possessed more positive effects on the wound healing process based on re-epithelialization and collagen formation than antibiotic treatment did.

Keywords: conditioned medium, diabetic induced rat, human umbilical cord mesenchymal stem cells, wound healing.

\section{Introduction}

Chronic wounds have become a global public health challenge. Unlike acute wounds, which heal without significant interventions, chronic wounds give major challenges to patients and doctors. There are varying etiologies of chronic wounds, and one of them is diabetes [1]. The prevalence of patients with diabetes worldwide is predicted to rise by $5.4 \%$ (300 million) by 2025 [2]. Ulcer in patients with diabetes is common, and diabetic foot ulcer in patients is a severe and complex case [3]. Diabetic foot ulcers

Copyright: Hendrawan, et al. Open Access. This article is distributed under the terms of the Creative Commons Attribution 4.0 International License (http://creativecommons.org/licenses/ by/4.0/), which permits unrestricted use, distribution, and reproduction in any medium, provided you give appropriate credit to the original author(s) and the source, provide a link to the Creative Commons license, and indicate if changes were made. The Creative Commons Public Domain Dedication waiver (http:// creativecommons.org/publicdomain/zero/1.0/) applies to the data made available in this article, unless otherwise stated. significantly influence the quality of life of patients, including limited and reduced mobility, diminished income, loss of job, and spending more to visit a physician or clinic for care [4]. Diabetes influences many aspects of life in patients, such as health, social, and economy. In the economic aspect, the cost of managing patients with diabetes with a lower extremity ulcer has a more economic burden than diabetic patients without ulcers [5]. Alternative solutions are needed to overcome the severe impacts that arise from diabetes, especially diabetic-related wounds. A diabetic wound is associated with decreased peripheral blood flow. Impaired angiogenesis and neovascularization result in insufficient oxygen and nutrient supply for the cells, leading to further impaired healing. Healing deficiency of diabetic wounds can be attributed to other factors, including decreased production of growth factors and reduced revascularization. A diabetic wound is challenging to treat and requires comprehensive 
procedures. The standard management of diabetic wounds includes surgical debridement, vascular assessment, infection treatment, and glycemic control. Even with holistic approaches, adjuvant therapies are still needed to improve healing times $[6,7]$.

Wound repair processes do not work smoothly in chronic wounds, like diabetic ulcers, because of several factors such as microbial infection [8], biofilm formation [9], and excessive inflammatory phase [10]. Recently, stem cells have been proven to have therapeutic potential. Mesenchymal stem cells (MSCs) are multipotent nonhematopoietic progenitor cells that show great promise for tissue regeneration. MSCs isolated from the umbilical cord and its conditioned medium (CM) can be easily obtained and refined compared to stem cells from other sources $[11,12]$. MSCs play a key role in three stages of the wound healing process and promote fibroblast migration during re-epithelialization [13]. The main concern in wound repair is inflammation. This concern can be solved by MSC characteristics that could reduce wound inflammation by repressing the proliferation of host T-cells [14,15]. The use of human umbilical cord-derived MSCs for tissue repair provides some advantages compared with other sources of MSCs, including cost-effectiveness, easy isolation process, low invasiveness, and low immunogenicity [16]. In addition, human umbilical cord-derived MSCs have other main characteristics, such as high proliferative ability and oxidative stress protein expression [17]. The previous studies demonstrated that umbilical cord MSCs have superior wound-healing capability either in an in vitro study [18] or in diabetic rats (in vivo study) [19]. Human umbilical cord MSCs (hUC-MSCs) were superior to fibroblasts in stimulating diabetic wound healing, marked by higher cell proliferation, collagen synthesis, and glycosaminoglycan level [20]. Another study also reported the efficacy of hUC-MSCs in stimulating cutaneous healing in burned rats [21]. Both UC-MSC and their CM were beneficial to diabetic wound healing, and in the context of wound healing application, CM is better than the cell itself [12]

As reported in a previous study, stem cells have essential roles in tissue regeneration conferred by its secreted paracrine factors, known as the secretome [22]. The secretome is defined as various molecules secreted from stem cells, such as cytokines, chemokines, and growth factors [23]. These molecules are secreted into the cell culture medium called CM [22]. Dressing and topical products, including topical antiseptic and antimicrobial application, are adjuvant therapies that are commonly used for diabetic foot ulcer care [3]. However, dressing and topical products might have limitations in curing diabetic foot ulcers. Furthermore, no studies have reported the efficacy comparison of hUC-MSC CM with antibiotics in wound repair.

Therefore, this study was conducted to evaluate the effect of hUC-MSC CM that was prepared under hypoxic conditions in stimulating rat fibroblast growth and collagen production (in vitro study) and to evaluate the treatment effect of hypoxic hUC-MSC $\mathrm{CM}$ on wound healing in diabetes-induced rats compared to antibiotic treatment (in vivo study).

\section{Materials and Methods}

\section{Ethical approval}

Animal experiments in this study were approved by Institutional Animal Care and Use Committees (IACUC) (001.KEPH/UPPM/FK/ IV/2019) of the Faculty of Medicine, Tarumanagara University. Surgical procedures for obtaining the fibroblast were conducted according to protocols approved by the Tarumanagara University IACUC, with IACUC approval number 003.KEPH/UPPM/FK/VI/2019.

\section{Study period and location}

The study was conducted from May 2019 to June 2020 at Tarumanagara Human Cell Technology Laboratory, Faculty of Medicine, Tarumanagara University, Indonesia.

\section{hUC-MSC CM preparation}

Human umbilical cord isolation

Fresh umbilical cords were collected after obtaining parental consent. The umbilical cords were washed with phosphate-buffered saline containing antibioticantimycotic $(\mathrm{Ab}-\mathrm{Am})$ solution before isolation. Veins and arteries were carefully removed from the umbilical cord. The Wharton jelly of the umbilical cord was then cut into small pieces and carefully placed on a $100-\mathrm{mm}$ culture dish, which was filled with cell culture medium consisting of alpha minimum essential medium ( $\alpha$ MEM; Gibco by Life Technologies, Grand Island, NY, USA) supplemented with 10\% fetal bovine serum (FBS; Gibco by Life Technologies) and 1\% Ab-Am (Gibco by Life Technologies). For $21 \mathrm{~d}$, MSC was migrated out from the umbilical cord tissue and harvested for further expansion.

\section{Identification of MSCS}

Immunophenotyping assay was conducted to ensure that the characteristic of the MSCs was in accordance with theInternational Society for Cell and Gene Therapy (ISCT) guidelines [24]. The surface markers CD73, CD105, and CD90, conjugated with phycoerythrin (R and D Systems), were used to stain MSCs. The assay used flow cytometry (FACS Calibur, BD, USA), and the analysis used CellQuest Pro software. The minimum acceptance criteria were 95\% for all positive markers [24].

\section{hUC-MSC CM production}

MSCs isolated from the umbilical cord were cultured until passage 6 (P6) in a T175 flask (Corning). The culture medium was deprived and replaced with basal medium with no supplement addition after reaching $70 \%-80 \%$ confluence and then incubated at $37^{\circ} \mathrm{C}$ in a $5 \% \mathrm{CO}_{2}$ incubator with two oxygen conditions: normoxic $\left(21 \% \mathrm{O}_{2}\right)$ and hypoxic $\left(5 \% \mathrm{O}_{2}\right)$. After $72 \mathrm{~h}$ of incubation, the hUC-MSC CM was collected and 
stored in a deep freezer $\left(-80^{\circ} \mathrm{C}\right)$ for long-term storage. hUC-MSCs were isolated and cultured at the Stem Cell and Cancer Institute Laboratory, Jakarta, Indonesia.

\section{Quantification of basic fibroblast growth factor} $(b F G F)$, vascular endothelial growth factor (VEGF), and pro-collagen 1 as wound healing-related paracrine factors

Enzyme-linked immunosorbent assay (ELISA) was performed to quantify paracrine factors secreted by MSCs related to wound healing, such as bFGF, VEGF, and pro-collagen 1 (R and D Systems). The procedure was performed according to the manufacturer's instructions, and the analysis was performed using four-parameter logistic software.

\section{In vitro study}

Rat fibroblast cell growth

Primary rat dermal fibroblasts were used for the in vitro study. MSCs (passage 5-6) cultured under hypoxic conditions for $72 \mathrm{~h}$ were used to produce the hUC-MSC CM. In addition, rat fibroblasts were obtained from donor rats. One male 12-weekold Sprague-Dawley rat (National Agency of Drug and Food Control, Jakarta) was used as a fibroblast donor. Fibroblasts were isolated from the rat's skin as described previously [25]. Fibroblasts at a density of $5 \times 10^{5}$ cells/well were seeded into 24 -well plates $(n=3)$ and then cultured in Dulbecco's modified Eagle medium (DMEM; Gibco by Life Technologies) supplemented with $10 \%(\mathrm{v} / \mathrm{v}) \mathrm{FBS}$ and $1 \mathrm{~mL}$ of penicillin/ streptomycin $(1 \times)$. hUC-MSC CM was added to the media culture with two concentrations, namely, $0.5 \%$ and $1 \%$. As a control, the same number of fibroblasts was seeded into wells and cultivated in the media without adding the hUC-MSC CM. Cells were incubated at $37^{\circ} \mathrm{C}$ in $5 \% \mathrm{CO}_{2}$ for $24 \mathrm{~h}$. The cell number was analyzed using the Cell Counting Kit-8 (CCK-8) viability assay (Sigma Aldrich, USA) and measured in a Multiskan reader at a wavelength $(\lambda)$ of $450 \mathrm{~nm}$ (Multiskan Ex, Thermo Scientific, USA).

\section{Analysis of collagen production on rat fibroblasts}

Collagen production by fibroblasts was measured using rat type I collagen ELISA kit (Elabscience Biotechnology Inc., China). Fibroblasts $\left(5 \times 10^{5}\right.$ cells/well $)$ were seeded into 24 -well plates, cultivated in Dulbecco's modified eagle medium (Gibco) supplemented with $10 \%(\mathrm{v} / \mathrm{v})$ FBS and $1 \mathrm{~mL}$ of penicillin/streptomycin $(1 \times)$, and incubated at $37^{\circ} \mathrm{C}$ in $5 \% \mathrm{CO}_{2}$ for $24 \mathrm{~h}$. The procedure was performed according to the manufacturer's instructions. The rat fibroblast collagen concentration was obtained using an ELISA reader at a wavelength $(\lambda)$ of $450 \mathrm{~nm}$.

\section{In vivo study}

Rat skin-wound healing model and induction of diabetes in rats

Nine adult male, 14-week-old Sprague-Dawley rats weighing 150-180 $\mathrm{g}$ were obtained from the
National Agency of Drug and Food Control, Jakarta, Indonesia. Before the experimental procedures, the animals were acclimatized to the laboratory conditions. They were allowed free access to standard laboratory food and water and housed individually in cages in a controlled environment $\left(23^{\circ} \mathrm{C} \pm 3^{\circ} \mathrm{C}\right.$, $30-70 \%$ humidity, and a 12:12 h light: dark cycle). For diabetes induction, the rats were rendered diabetic 1 week before treatment by a single-dose intraperitoneal injection of streptozotocin (STZ) $(50 \mathrm{mg} / \mathrm{kg}$ body weight; Selleck Chemicals). Blood glucose was checked $48 \mathrm{~h}$ post-injection, and blood samples were obtained from the tail vein of the animals for glucose assay. The rats were considered diabetic if three days after the STZ injection, the non-fasting blood glucose was more than $200 \mathrm{mg} / \mathrm{dL}$ and presented with at least 3 days of persistent hyperglycemia.

The diabetes-induced rats were randomized and divided into the following three treatment groups $(n=3)$ : Which were non-treatment (without any medication), antibiotics (topical medication with bactoderm mupirocin ointment $2 \%$, Ikapharmindo Putramas, Indonesia), and hUC-MSC CM groups (intradermal injection with $0.5 \mathrm{~mL}$ of undiluted $\mathrm{CM}$ on a peripheral wound). For the experimental procedure, the rats were weighed and anesthetized by intraperitoneal administration of ketamine 10\% INJ (Kepro B.V, Holland; 40-80 mg/kg body weight) and xylazine2\% (Interchemie werken "De Adelaar" B.V. Metaalweg 8 Venray, Holland; $5-10 \mathrm{mg} / \mathrm{kg}$ body weight). Then, the hair on the backside was shaved and disinfected. The skin on the dorsal area of the rats was punctured using an 8-mm skin punch, with each rat having two ulcer wounds and receiving different treatments. After the procedure, wounds were closed with Tegaderm (3M Nexcare) and fixed with a suture at 3-4 points. Hypafix (Essity, Healthcare 21 Group, Ref. 71442-03) was added to secure the Tegaderm. Three wounds per treatment were analyzed in this study. The wound observations, photos, and measurements were conducted at three endpoints: 0,7 , and 14 $\mathrm{d}$ post-skin puncture. The percentage of wound closure was calculated using the equation: percentage of wound closure $(\%)=\left(A_{0}-A_{t}\right) / A_{0} \times 100 \%$, where $A_{0}$ is the wound area at Day 0 and $A_{t}$ is the wound area at 7 or $14 \mathrm{~d}$ post-skin puncture.

\section{Histopathological analysis}

All Sprague-Dawley rats were euthanized at $14 \mathrm{~d}$ post-skin puncture. Skin specimens from the wound site were dissected and collected. Specimens were fixed in $10 \%$ formalin and sent to the Pathology and Anatomy Laboratory, Primate Research Center, Bogor Agricultural University, for further examinations, including re-epithelialization with hematoxylin and eosin (HE) staining and collagen formation area measurement with Masson's trichrome staining. HE staining and MT staining were performed according to Sheehan and Hrapchak [26]. Tissue sections 
were observed and captured using a Nikon Eclipse $80 \mathrm{i}$ microscope to evaluate re-epithelialization and collagen-deposited areas. Furthermore, the width of re-epithelialization and collagen-deposited areas was analyzed using the ImageJ software.

\section{Statistical analysis}

All data were presented as mean $\pm \mathrm{SD}$. The differences between the two independent groups were analyzed by Mann-Whitney and Kruskal-Wallis tests to analyze the differences among the three independent groups. Statistical analysis was conducted using SPSS v.23, with a $95 \%$ confidence interval. Values were considered statistically significant at $\mathrm{p}<0.05$.

\section{Results}

\section{hUC-MSC characteristics}

The human MSCs obtained from umbilical cord primary isolation were $1 \times 10^{5}$ cells and expanded from passage $0-6$. Culture expansion used a cell density of $7 \times 10^{3}$ cells $/ \mathrm{cm}^{2}$ as the seeding concentration standard. hUC-MSC morphology was like a spindle-shaped fibroblast (Figure-1), and the size of the MSC was approximately $120 \mu \mathrm{m}$. There was no significant change shown by MSCs based on their morphology throughout culturing.

MSCs expressed markers CD73, CD90, and CD105 and did not express hematopoietic markers such as CD34, CD45, CD14, and CD19. The flow cytometry results confirmed that the cells used were MSCs, with values of all positive markers above 95\% and negative markers under 2\% (Table-1). The

Table-1: Marker analysis of obtained mesenchymal stem cells (MSCs) from human umbilical cord.

\begin{tabular}{lc}
\hline Markers & Population percentage \\
\hline CD105 & 99.47 \\
CD73 & 99.26 \\
CD90 & 99.52 \\
CD14 & 0 \\
CD45 & 0.26 \\
CD19 & 1.47 \\
\hline
\end{tabular}

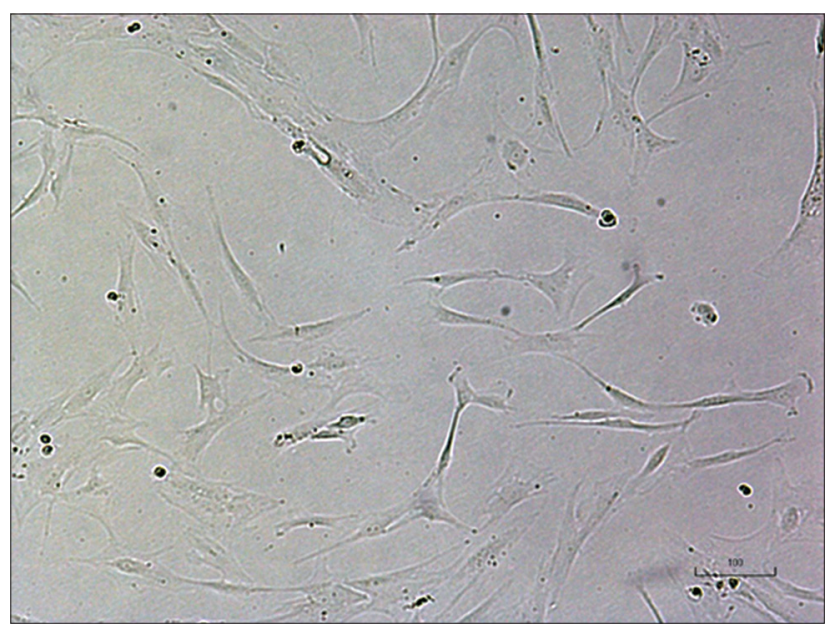

Figure-1: Microscopic Appearance of human umbilical cord mesenchymal stem cells (passage 5) with magnification $400 \times$. Scale bar: $100 \mu \mathrm{m}$. surface epitopes are considered MSC-specific markers according to the ISCT [24].

\section{Pro-collagen, VEGF, and bFGF Content in hUC-MSC CM}

We used passage six cells for CM production because of their defined characters of MSC. The MSC cells were cultured with MEM-Alpha without an additional growth supplement after reaching $70-80 \%$ confluence. The MSC cells were further incubated for $72 \mathrm{~h}$ to allow the secretion of the secretome. To investigate the favorable condition of secretome secretion, we placed the MSCs under two conditions (normoxic $\left[21 \% \mathrm{O}_{2}\right]$ and hypoxic $\left[5 \% \mathrm{O}_{2}\right]$ ). The CM was stored in a deep freezer to maintain the stability of its protein content for long-term storage. On the basis of the ELISA results of CM from normoxic and hypoxic hUC-MSC, it can be concluded that there were concentration differences for all types of secreted protein between the two conditions (Figure-2). In CM from hUC-MSC hypoxic conditions, VEGF, bFGF, and pro-collagen 1 concentrations were higher than those in normoxic conditions. Furthermore, in CM from the hUC-MSC normoxic conditions, VEGF was not secreted, whereas, in CM from the hypoxic condition, it was secreted more than $2 \mathrm{ng} / \mathrm{mL}$. In line with these results, bFGF and pro-collagen 1 secretions were highly secreted under hypoxic conditions. These results indicate that the incubation of hUC-MSCs under hypoxic conditions was stimulated to secrete more VEGF, bFGF, and pro-collagen 1. CM from hypoxic hUC-MSC was used in further in vitro and in vivo trials based on these results.

\section{Cell growth of rat fibroblast}

Fibroblasts were treated with two concentrations of hypoxic hUC-MSC CM $(0.5 \%$ and $1 \%)$ to observe cell growth and function. The cell number of fibroblasts treated with CM $1 \%$ at $24 \mathrm{~h}$ after cell seeding was higher than the cell numbers of those treated with CM $0.5 \%$ and untreated. However, the mean cell number of the three groups did not differ significantly (control group: $1.34 \times 10^{6} \pm 2.83 \times 10^{4}$; CM $0.5 \%$ group: $1.38 \times 10^{6} \pm 3.38 \times 10^{4}$; CM $1 \%$ group: $\left.1.40 \times 10^{6} \pm 1.02 \times 10^{5}\right) \quad$ (Figure-3a). Although the results showed that a higher concentration of hypoxic

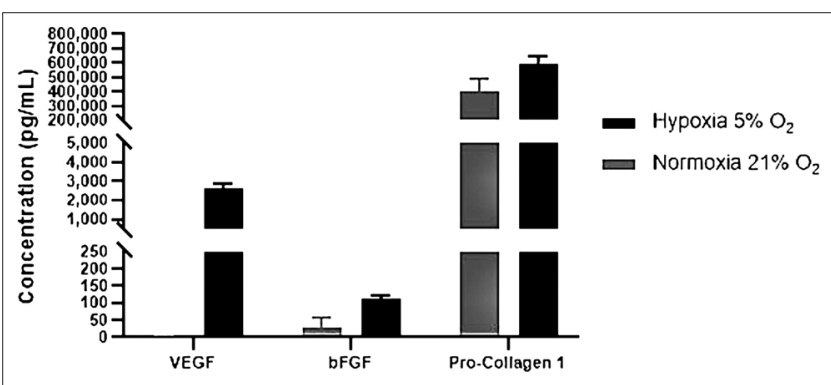

Figure-2: Vascular endothelial growth factor, basic fibroblast growth factor, and pro-collagen 1 amount produced from human umbilical cord mesenchymal stem cells cultured in normoxic and hypoxic conditions. Data show mean \pm SD. 


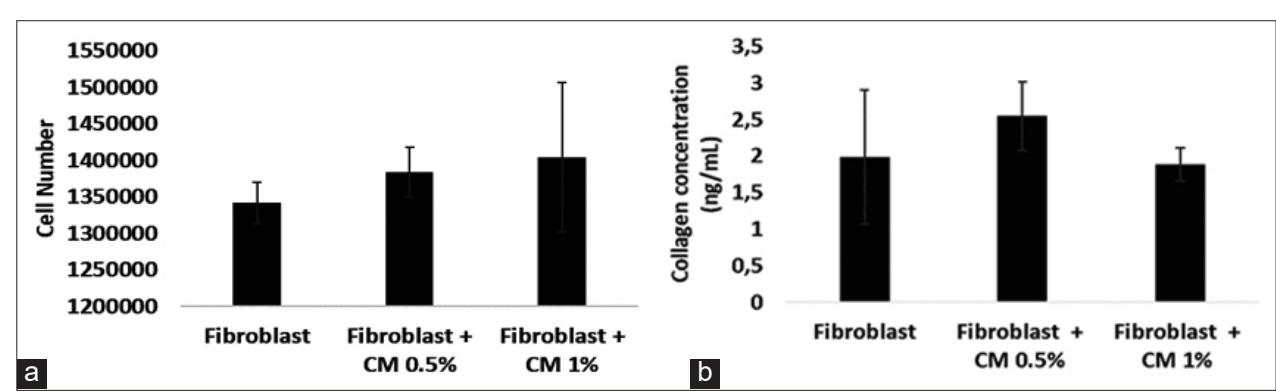

Figure-3: (a) Cell number of fibroblasts treated with two different concentrations of conditioned medium (CM) and (b) collagen concentration secreted from fibroblast post-treated with human umbilical cord mesenchymal stem cells CM $0.5 \%$ and $1 \%$ concentrations $(n=3)$. Data show mean \pm SD.

hUC-MSC CM might better facilitate fibroblast growth, statistically, there was no significant difference in the cell number of the fibroblast (Figure-3a). These results showed that adding a certain concentration of CM did not significantly affect fibroblast cell growth.

\section{Collagen secretion}

To examine the effect of adding hypoxic hUCMSC CM against fibroblast function in producing collagen, we also used two concentrations of CM. Hypoxic hUC-MSC CM (both $0.5 \%$ and $1 \%$ ) showed improved collagen secretion than the control group did. However, the mean collagen concentration was not significantly different between groups (control group: $1.99 \pm 0.91 \mathrm{ng} / \mathrm{mL}$; CM $0.5 \%$ group: $2.55 \pm 0.47 \mathrm{ng} / \mathrm{mL}$; CM $1 \%$ group: $1.89 \pm 0.23 \mathrm{ng} / \mathrm{mL}$ ) (Figure-3b). Our study may indicate that collagen production did not strongly depend on CM, and the lower or higher concentration of CM did not significantly increase collagen production.

\section{Wound healing observation in diabetic ulcer}

The wound healing process was observed by re-epithelialization and newly synthesized collagen. In addition, different responses were observed between the non-treatment, antibiotic, and hypoxic hUC-MSC $\mathrm{CM}$ groups. Our preliminary experiment showed that CM obtained from hypoxic hUC-MSC showed a superior effect in wound healing compared with that from normoxic hUC-MSC. On the basis of these findings, we treated the wound with CM of MSCs cultivated under hypoxic conditions. As hypothesized, the hypoxic hUC-MSC CM demonstrated wound healing potential in diabetic animal models, both in macroscopic (Figure-4a) and microscopic observations (Figure-5).

At the macroscopic observation, the hypoxic hUC-MSC CM group demonstrated faster wound closure post-skin puncture. Figure- $4 \mathrm{~b}$ showed the highest wound closure percentage on wounds treated with hypoxic hUC-MSC CM $(37.5 \% \pm 0 \%)$ after $7 \mathrm{~d}$ post-skin puncture compared with the antibiotic $(20.83 \% \pm 19.09 \%)$ and non-treatment groups $(20.83 \% \pm 19.09 \%)$. The hypoxic hUC-MSC CM treated wound showed better and faster wound closure than that treated with an antibiotic and untreated wound at $7 \mathrm{~d}$ post-skin puncture. The hypoxic hUC-MSC CM group had the same wound closure percentage $(75 \% \pm 12.5 \%)$ as that with the antibiotic group $(75 \% \pm 0 \%)$ and had a higher wound closure percentage than that with the non-treatment group $(62.5 \% \pm 12.5 \%)$ at $14 \mathrm{~d}$ post-skin puncture (Figure-4a). The wound closure percentage in wounds treated with hypoxic hUC-MSC CM was the same as that of the antibiotic group at $14 \mathrm{~d}$, but still higher than that of the non-treatment group (Figure-4b).

The hypoxic hUC-MSC CM group exhibited the largest re-epithelialization area for microscopic observation compared with the antibiotic group (Figure-6a). Moreover, there was no re-epithelialization area found in the non-treatment group. In addition, the epithelium length $(2806 \pm 338 \mu \mathrm{m})$ of the hypoxic UC-MSC CM group was 2.15-fold longer than the epithelium length of the antibiotic group $(1303 \pm 940 \mu \mathrm{m})$ (Figure-6a). These results were also supported by the width of the collagen formation area data (Figure-6b). Correspondingly, the area of the newly generated collagen tissue in the hUC-MSC CM group $\left(29.03 \times 10^{4} \pm 60.99 \times 10^{4} \mu \mathrm{m}^{2}\right)$ was five-fold wider than that in the antibiotic group $\left(57.93 \times 10^{4} \pm 64.77 \times 10^{4} \mu^{2}\right)$ (Figure-6b). Altogether, these findings demonstrated that hypoxic hUC-MSC $\mathrm{CM}$ treatment showed a distinct effect to facilitate wound repair in our diabetic wound model.

\section{Discussion}

This study analyzed the efficacy of hypoxic hUC-MSC CM on wound healing of diabetic rats. We also performed antibiotic treatment for comparison. First, we confirmed that our cells were MSCs based on morphology observation and supported by marker analysis results (Figures-1 and 2). To investigate the suitable conditions to produce $\mathrm{CM}$, we observed two MSC-cultivated conditions (normoxic and hypoxic conditions). Interestingly, the production levels of VEGF, bFGF, and pro-collagen 1 were different. The hypoxic condition increased the production of the CM by MSCs. It has been reported that the mRNA level and protein expression of VEGF-A were higher in MSCs prepared under hypoxic conditions compared with normoxic conditions [27]. Another study by Page et al. [28] also reported that gene expression 


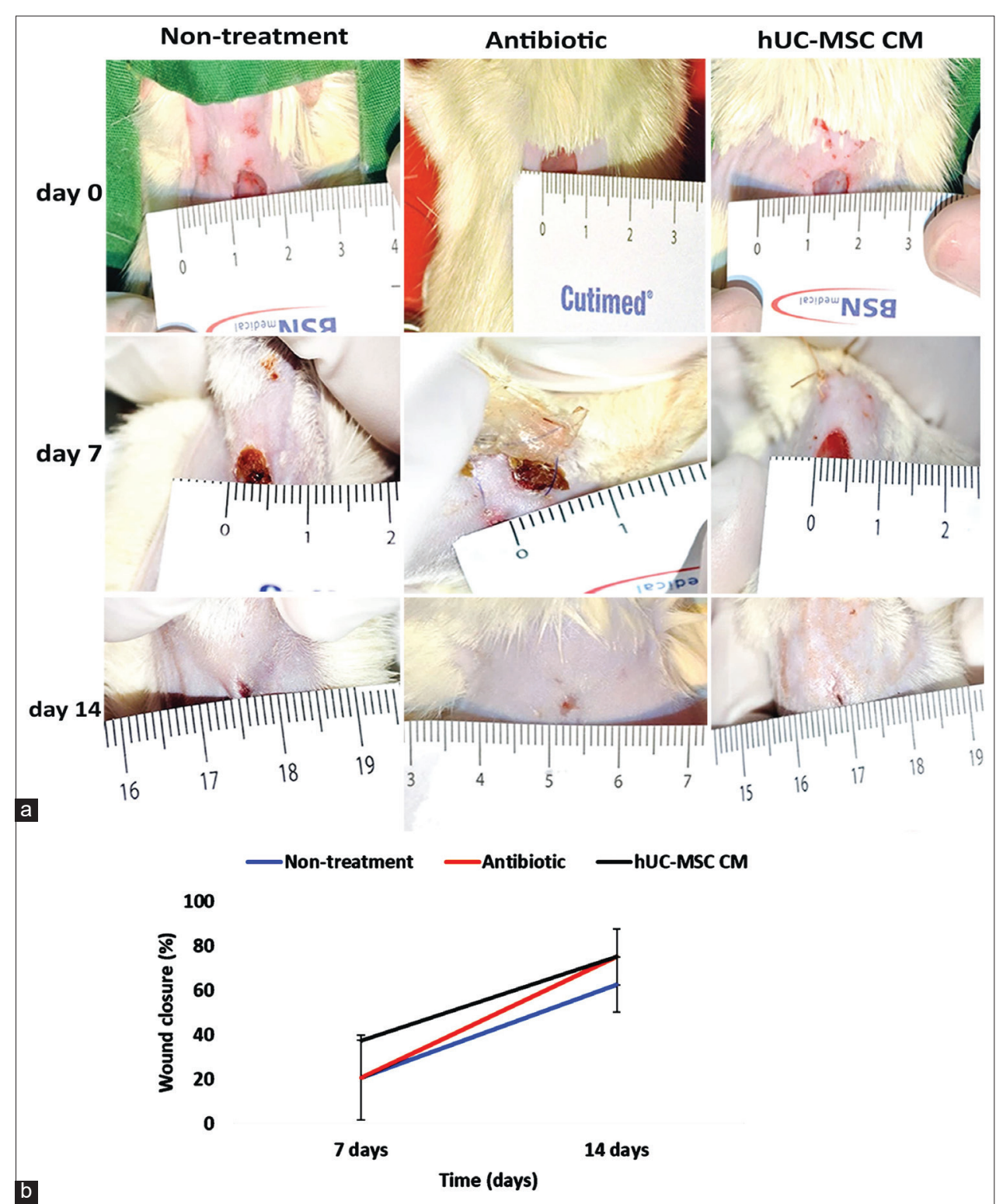

Figure-4: (a) Wound closure measurement on diabetic induced rats (non-treatment, antibiotic and human umbilical cord mesenchymal stem cells conditioned medium groups) at three endpoints (0-, 7-, and 14-days). (b) Percentage of wound closure comparison between groups at 7- and 14-days post-skin puncture.

and paracrine secretion are strongly affected by serum and oxygen concentration. Our study observed that there was no VEGF production by the MSCs under normoxic conditions. In contrast to our findings, Chen et al. [29] investigated that VEGF-A was still produced at a lower concentration under normoxic conditions by bone marrow MSCs than under hypoxic conditions. However, our result in bFGF was supported by their findings. Meanwhile, we found that pro-collagen 1 was secreted in these two setup conditions, and their concentrations were slightly different. These data suggested that hypoxic conditions were preferable to stimulate the secretion of secretome/ paracrine. Similar to our finding, Hsiao et al. [30] also demonstrated that hypoxic conditions favored VEGF-A and ANG production as angiogenic factors. Likewise, a previous study reported that VEGF and bFGF induce angiogenesis in wounded skin [31].
VEGF acts on wound repair through angiogenesis, collagen synthesis, and epithelialization [32].

To explore the effect of hypoxic hUC-MSC $\mathrm{CM}$ on fibroblast cell growth and collagen secretion in vitro, we treated fibroblast with hypoxic hUCMSC CM with two concentrations $(0.5 \%$ and $1 \%)$. Our findings showed that hypoxic hUC-MSC CM increased the number of fibroblasts, particularly in the group treated with a higher concentration (1\%). Thus, it suggested that hypoxic hUC-MSC CM addition exerted a positive effect on fibroblast proliferation. Kim et al. [33] reported a similar result, and they investigated the highest growth of HDF, cell concentration, and protein level observed in the USC-CM group. They also evaluated the activity of USC-MSC to induce migration of HDF compared with HDF and another MSC-CM group. Other studies also proved that the application of MSC-CM 
facilitated higher fibroblast proliferation than those in the control group [34].

Our findings showed that adding hypoxic hUCMSC CM enhances collagen secretion, especially in fibroblasts treated with hypoxic hUC-MSC CM $0.5 \%$. It may indicate that the collagen production did not strongly depend on the concentration of used hypoxic hUC-MSC CM. The previous studies by Kim et al. [33] demonstrated that the production of ECM (collagen, elastin, and fibronectin) was also triggered by USC-CM. Villanueva et al. [35] examined a lower collagen production from lung fibroblasts found in the control group (without $\mathrm{CM}$ addition) compared with adding endothelial cell-derived CM $(1: 10)$ at a 6-h incubation. At 24-h incubation, treatment with an increasing concentration of $\mathrm{CM}$ elevated the amount of collagen synthesized. As one of the extracellular matrix compositions in the skin, collagen plays a key role in growth and determines skin's elasticity [36].

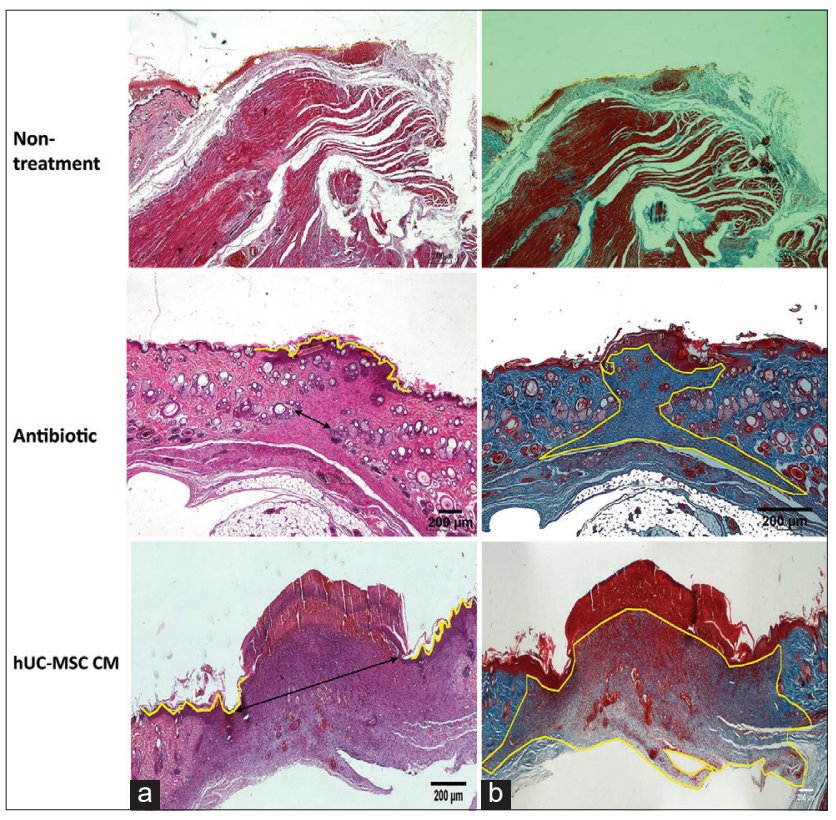

Figure-5: Histopathological analysis of (a) re-epithelialization using Hematoxylin and Eosin staining and (b) collagen formation using Masson's trichrome staining on the wound site of diabetic induced rats. Re-epithelialization and collagen formation areas are indicated with yellow lines. Scale bar $200 \mu \mathrm{m}$ in all panels. The double-headed arrows point the edge of the scar.
To investigate the therapeutic effect of hypoxic hUC-MSC CM in vivo, we provided an antibiotic group for comparison and the non-treatment as a control group. We chose topical antibiotic treatment because of its ability to inhibit or kill microbes on an open wound. Our results showed that the hypoxic hUC-MSC CM group had a beneficial effect on the wound healing process, which was revealed by macroscopic and microscopic observation. An expected outcome was a reduction of the wound surface area. Although not statistically significant, the hUC-MSC CM group demonstrated faster wound closure since observation at $7 \mathrm{~d}$ post-skin puncture. Non-significant results might be caused by the small sample size and the length of the observation time. The created wound site was reduced at $14 \mathrm{~d}$ post-wounding. The hypoxic hUC-MSC CM group had the same wound closure percentage as that of the antibiotic group and had a higher wound closure percentage than that of the non-treatment group at $14 \mathrm{~d}$ post-skin puncture. A study by Han et al. [19] showed a wound healing process that was significantly lower in diabetic rats than in normal rats at days 7 and 14. This phenomenon in their study showed that blockade of the Wnt signaling pathway slowed the healing of skin wounds in diabetic rats. A study by Assi et al. [37] in mice showed that wounds treated with topical scaffolds containing MSCs increased proliferation without increasing apoptosis and VEGF-positive cells. Another study by Zhou et al. [38] demonstrated that hUC-MSC CM/ hydrogel promoted wound closure and constricted fibrotic and hypertrophic scar tissue formation.

The efficacy of antibiotics to facilitate wound healing was not as high as that triggered by hUC-MSC $\mathrm{CM}$ application but was better than the non-treatment group. Reports from Padeta et al. [34] demonstrated a similar finding to our results. They found that the MSC $\mathrm{CM}$ showed a prominent effect in promoting wound healing than bioplacenton application as their control group prepared from bovine placenta extract $10 \%$, neomycin sulfate $0.5 \%$, and gel base. Thus, besides the self-renewal capacity, human MSCs and their secretome may have antimicrobial activity. Our hypothesis was supported by Krasnodembskaya et al. [39], who observed the antimicrobial efficacy of human

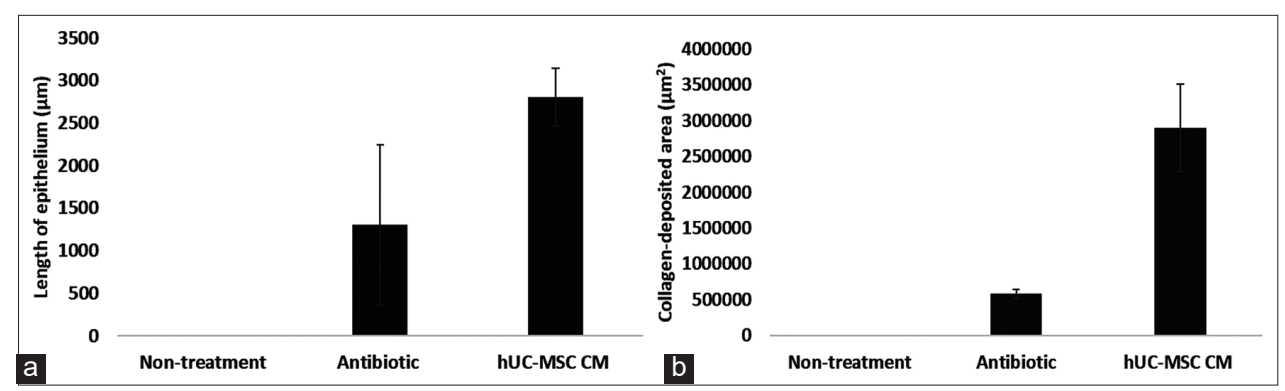

Figure-6: (a) Length of epithelium and (b) width of collagen-deposited area comparison between non-treatment, antibiotic, and human umbilical cord mesenchymal stem cells conditioned medium group in diabetic induced rats at 14 days post skin punctures. 
MSCs and their CM. This antimicrobial activity was exerted by the antimicrobial peptide LL-37 production. These results suggest that using hUC-MSC CM had a superior effect compared with antibiotics application alone. These benefits might trigger the efficacy of hypoxic hUC-MSC CM.

Wound healing can be characterized by re-epithelialization [40]. As a further investigation, we performed the histopathological analysis of our specimen to observe the effect of given treatments on wound repair. We observed and measured the newly formed epithelial tissue and collagen-deposited areas in hypoxic hUC-MSC CM and the topical antibiotic group. It indicated that the treated skin was at the proliferation phase. Furthermore, the proliferation phase in wound repair is marked by four processes, including re-epithelialization, angiogenesis, collagen synthesis, and extracellular formation $[41,42]$. In re-epithelialization, the new epithelium formed by proliferation, migration, and keratinocyte differentiation also protects beneath the epidermal layer [43].

Histopathological analysis of a wound site showed an increase in re-epithelialization area in the hypoxic hUC-MSC CM group compared with the non-treatment and antibiotic groups at $14 \mathrm{~d}$ post-skin puncture. A similar study reported by Shrestha et al. [12] showed enhanced re-epithelialization after hUC-MSC CM application on the wound at $14 \mathrm{~d}$ post-treatment [9]. Consistent with the re-epithelialization result, we confirmed the largest collagen deposition on the hypoxic hUC-MSC CM group $14 \mathrm{~d}$ post-skin puncture. Correspondingly, Padeta et al. [34] reported MSC-CM accelerated collagen deposition in wounded rats compared with the control group. In addition, Li et al. [44] found a pronounced effect of MSC CM on keratinocyte proliferation and migration in a high-glucose microenvironment.

\section{Conclusion}

Hypoxia was identified as a better condition to enhance VEGF, bFGF, and pro-collagen 1 secretion. We proved the ability of the hypoxic hUC-MSC CM to facilitate fibroblast cell growth and collagen synthesis through the in vitro study. CM from hypoxic hUC-MSC possessed positive and better wound healing effects than antibiotic application on the wound. In addition, wound healing mediated by the hypoxic hUC-MSC CM was based on re-epithelialization and collagen formation evidence in the in vivo study. Our findings collectively confirmed that hUC-MSC CM may be used as an alternative treatment to cope with diabetes-related wounds. Our findings might encourage clinical studies that analyze the benefits of hUCMSC CM for diabetic patients with ulcers.

\section{Authors' Contributions}

SH, STT, and YK: Conceived, designed, and managed the study. SH and STT: Performed sample collections. YK, CAL, and DF: Prepared human umbilical cord mesenchymal stem cell secretome. $\mathrm{SH}$, JL, and BSM: Did data collection and analysis. SH, EB, BSM, HUB, and STT: Drafted the manuscript. All authors participated in the revision of this manuscript and approved the submission.

\section{Acknowledgments}

The authors are thankful to Pathology and Anatomy Laboratory, Primate Research Center, Bogor Agricultural University, Indonesia, for helping us in histopathological analysis. We also thank Nuraeni for capturing and preparing the figures. The study was funded by a grant from Tarumanagara University, Indonesia (Grant Number: 012 Int-DIR. PPKM/UNTAR/I/2018).

\section{Competing Interest}

The authors declare that they have no competing interests.

\section{Publisher's Note}

Veterinary World remains neutral with regard to jurisdictional claims in published institutional affiliation.

\section{References}

1. Demidova-Rice, T.N., Hamblin, M.R. and Herman, I.M. (2012) Acute and impaired wound healing. Adv. Skin Wound Care, 25(7): 304-314.

2. King, H., Aubert, R.E. and Herman, W.H. (1998) Global burden of diabetes, 1995-2025: Prevalence, numerical estimates, and projections. Diabetes Care, 21(9): 1414-1431.

3. Reiber, G., Lipsky, B. and Gibbons, G. (1998) The burden of diabetic foot ulcers. Am. J. Surg., 176(2A Suppl): 5S-10S.

4. Goodridge, D., Trepman, E. and Embil, J.M. (2005) Healthrelated quality of life in diabetic patients with foot ulcers: Literature review. J. Wound Ostomy Cont. Nurs., 32(6): 368-377.

5. Driver, V.R., Fabbi, M., Lavery, L.A. and Gibbons, G. (2010) The costs of diabetic foot: The economic case for the limb salvage team. J. Vasc. Surg., 52(Suppl 3): 17S-22S.

6. Schreml, S. and Berneburg, M. (2017) The global burden of diabetic wounds. Br. J. Dermatol., 176(4): 845-846.

7. Everett, E. and Mathioudakis, N. (2018) Update on management of diabetic foot ulcers. Ann. N. Y. Acad. Sci., 1411(1): 153-165.

8. Edwards, R. and Harding, K.G. (2004) Bacteria and wound healing. Curr. Opin. Infect. Dis., 17(2): 91-96.

9. Wolcott, R.D., Rhoads, D.D. and Dowd, S.E. (2008) Biofilms and chronic wound inflammation. J. Wound Care, 17(8): 333-341.

10. Eming, S.A., Krieg, T. and Davidson, J.M. (2007) Inflammation in wound repair: Molecular and cellular mechanisms. J. Invest. Dermatol., 127(3): 514-525.

11. Lee, C., Shim, S., Jang, H., Myung, H., Lee, J., Bae, C.H., Myung, J.K., Kim, M.J., Lee, S.B. and Jang, W.S. (2017) Human umbilical cord blood-derived mesenchymal stromal cells and small intestinal submucosa hydrogel composite promotes combined radiation-wound healing of mice. Cytotherapy, 19(9): 1048-1059.

12. Shrestha, C., Zhao, L., Chen, K., He, H. and Mo, Z. (2013) Enhanced healing of diabetic wounds by subcutaneous administration of human umbilical cord derived stem cells and their conditioned media. Int. J. Endocrinol., 2014: 1-10.

13. Maxson, S., Lopez, E.A., Yoo, D., DanilkovitchMiagkova, A. and LeRoux, M.A. (2012) Concise review: 
Role of mesenchymal stem cells in wound repair. Stem Cells Transl. Med., 1(2): 142-149.

14. Nuschke, A. (2014) Activity of mesenchymal stem cells in therapies for chronic skin wound healing. Organogenesis, 10(1): 29-37.

15. Beyth, S., Borovsky, Z., Mevorach, D., Liebergall, M., Gazit, Z., Aslan, H., Galun, E. and Rachmilewitz, J. (2005) Human mesenchymal stem cells alter antigen-presenting cell maturation and induce T-cell unresponsiveness. Blood, 105(5): 2214-2219.

16. Hoffmann, A., Floerkemeier, T., Melzer, C. and Hass, R. (2017) Comparison of in vitro-cultivation of human mesenchymal stroma/stem cells derived from bone marrow and umbilical cord. J. Tissue Eng. Regen. Med., 11(9): 2565-2581.

17. Zazzeroni, L., Lanzoni, G., Pasquinelli, G. and Ricordi, C. (2017) Considerations on the harvesting site and donor derivation for mesenchymal stem cells-based strategies for diabetes. CellR4 Repair Replace. Regen. Reprogram., 5(5): e2435.

18. You, H.J., Namgoong, S., Han, S.K., Jeong, S.H., Dhong, E.S. and Kim, W.K. (2015) Wound-healing potential of human umbilical cord blood-derived mesenchymal stromal cells in vitro a pilot study. Cytotherapy, 17(11): 1506-1513.

19. Han, Y., Sun, T., Han, Y., Lin, L., Liu, C., Liu, J., Yan, G., and Tao, R. (2019) Human umbilical cord mesenchymal stem cells implantation accelerates cutaneous wound healing in diabetic rats via the Wnt signaling pathway. Eur. J. Med. Res., 24(10): 1-9.

20. Jung, J., Yoon, Y., Lee, H., Kang, S. and Han, S. (2018) Comparison of human umbilical cord blood-derived mesenchymal stem cells with healthy fibroblasts on wound-healing activity of diabetic fibroblasts. Int. Wound J., 15(1): 133-139.

21. Liu, L., Yu, Y., Hou, Y., Chai, J., Duan, H., Chu, W., Zhang, H., Hu, Q. and Du, J. (2014) Human umbilical cord mesenchymal stem cells transplantation promotes cutaneous wound healing of severe burned rats. PLoS One, 9(2): e88348.

22. Xu, Y., Guo, S., Wei, C., Li, H., Chen, L., Yin, C. and Zhang, C. (2016) The comparison of adipose stem cell and placental stem cell in secretion characteristics and in facial antiaging. Stem Cells Int., 2016: 7315830.

23. Park, S.R., Kim, J.W., Jun, H.S., Roh, J.Y., Lee, H.Y. and Hong, I.S. (2018) Stem cell secretome and its effect on cellular mechanisms relevant to wound healing. Mol. Ther., 26(2): 606-617.

24. Dominici, M., Le Blanc, K., Mueller, I., SlaperCortenbach, I., Marini, F.C., Krause, D.S., Deans, R.J., Keating, A., Prockop, D.J. and Horwitz, E.M. (2006) Minimal criteria for defining multipotent mesenchymal stromal cells. The international society for cellular therapy position statement. Cytotherapy, 8(4): 315-317.

25. Hendrawan, S., Bono, E., Hutter, A., Weber, U., Lheman, J. and Baer, H.U. (2020) Evaluation of 3D PLLA scaffolds coated with nano-thick collagen as carrier for hepatocytes. J. Biomed. Mater. Res. B Appl. Biomater., 109(5): 723-732.

26. Sheehan, D.C. and Hrapchak, B.B. (1980) Theory and Practice of Histotechnology. Vol. 2. Mosby, Maryland Heights, Missouri. p101-120.

27. Bartaula-Brevik, S., Bolstad, A.I., Mustafa, K. and Pedersen, T.O. (2017) Secretome of mesenchymal stem cells grown in hypoxia accelerates wound healing and vessel formation in vitro. Int. J. Stem. Cell Res. Ther., 4(1): 1-9.

28. Page, P., DeJong, J., Bandstra, A. and Boomsma, R.A. (2014) Effect of serum and oxygen concentration on gene expression and secretion of paracrine factors by mesenchymal stem cells. Int. J. Cell Biol., 2014: 601063

29. Chen, L., Xu, Y., Zhao, J., Zhang, Z., Yang, R., Xie, J., Liu, X. and Qi, S. (2014) Conditioned medium from hypoxic bone marrow-derived mesenchymal stem cells enhances wound healing in mice. PLoS One., 9(4): e96161.

30. Hsiao, S.T., Lokmic, Z., Peshavariya, H., Abberton, K.M., Dusting, G.J., Lim, S.Y. and Dilley, R.J. (2013) Hypoxic conditioning enhances the angiogenic paracrine activity of human adipose-derived stem cells. Stem Cells Dev., 22(10): 1614-1623.

31. Nissen, N.N., Polverini, P., Koch, A.E., Volin, M.V., Gamelli, R.L. and Dipietro, L.A. (1998) Vascular endothelial growth factor mediates angiogenic activity during the proliferative phase of wound healing. Am. J. Pathol., 152(6): 1445.

32. Stojadinovic, O. (2007) A novel non-angiogenic mechanism of VEGF: Stimulation of keratinocyte and fibroblast migration. Wound Repair Regen., 15(2): A30.

33. Kim, Y.J., Seo, D.H., Lee, S.H., Lee, S.H., An, G.H., Ahn, H.J., Kwon, D., Seo, K.W. and Kang, K.S. (2018) Conditioned media from human umbilical cord blood-derived mesenchymal stem cells stimulate rejuvenation function in human skin. Biochem. Biophys. Rep., 16(5): 96-102.

34. Padeta, I., Nugroho, W.S., Kusindarta, D.L., Fibrianto, Y.H. and Budipitojo, T. (2017) Mesenchymal stem cell-conditioned medium promote the recovery of skin burn wound. Asian J. Anim. Vet. Adv., 12(3): 132-141.

35. Villanueva, A.G., Farber, H.W., Rounds, S. and Goldstein, R.H. (1991) Stimulation of fibroblast collagen and total protein formation by an endothelial cell-derived factor. Circ. Res., 69(1): 134-141.

36. Fulop, T., Khalil, A. and Larbi, A. (2012) The role of elastin peptides in modulating the immune response in aging and age-related diseases. Pathol. Biol., 60(1): 28-33.

37. Assi, R., Foster, T.R., He, H., Stamati, K., Bai, H., Huang, Y., Hyder, F., Rothman, D., Shu, C. and HomerVanniasinkam, S. (2016) Delivery of mesenchymal stem cells in biomimetic engineered scaffolds promotes healing of diabetic ulcers. Regen. Med., 11(3): 245-260.

38. Zhou, P., Li, X., Zhang, B., Shi, Q., Li, D. and Ju, X. (2019) A human umbilical cord mesenchymal stem cell-conditioned medium/chitosan/collagen/ $\beta$-glycerophosphate thermosensitive hydrogel promotes burn injury healing in mice. Biomed Res. Int., 2019(2): 5768285.

39. Krasnodembskaya, A., Song, Y., Fang, X., Gupta, N., Serikov, V., Lee, J. and Matthay, M.A. (2010) Antibacterial effect of human mesenchymal stem cells is mediated in part from secretion of the antimicrobial peptide LL-37. Stem Cells, 28(12): 2229-2238.

40. Pastar, I., Stojadinovic, O., Yin, N.C., Ramirez, H., Nusbaum, A.G., Sawaya, A., Patel, S.B., Khalid, L., Isseroff, R.R. and Tomic-Canic, M. (2014) Epithelialization in wound healing: A comprehensive review. Adv. Wound Care, 3(7): 445-464

41. Guo, S. and Dipietro, L.A. (2010) Factors affecting wound healing. J. Dent. Res., 89(3): 219-229.

42. Mathieu, D., Linke, J.C. and Wattel, F. (2006) Non-healing wounds. In: Handbook on Hyperbaric Medicine. Springer, Dordrecht. p401-428.

43. Krishnaswamy, V.R. and Korrapati, P.S. (2014) Role of dermatopontin in re-epithelialization: Implications on keratinocyte migration and proliferation. Sci. Rep., 4(7385): 1-10.

44. Li, M., Zhao, Y., Hao, H., Dai, H., Han, Q., Tong, C., Liu, J., Han, W. and Fu, X. (2015) Mesenchymal stem cell-conditioned medium improves the proliferation and migration of keratinocytes in a diabetes-like microenvironment. Int. J. Low. Extrem. Wounds, 14(1): 73-86. 